\title{
Scottish Primary School Children Who Consume Greater Levels of Fruit and Vegetables Have Improved Health Markers
}

\author{
Heba Althubaiti*, Madeline Coleman \\ Institute of Biological and Environmental Sciences, University of Aberdeen, Aberdeen, UK \\ Email: *r01haa13@abdn.ac.uk
}

How to cite this paper: Althubaiti, H. and Coleman, M. (2017) Scottish Primary School Children Who Consume Greater Levels of Fruit and Vegetables Have Improved Health Markers. Food and Nutrition Sciences, 8, 485-511.

https://doi.org/10.4236/fns.2017.85034

Received: April 6, 2017

Accepted: May 14, 2017

Published: May 17, 2017

Copyright $\odot 2017$ by authors and Scientific Research Publishing Inc. This work is licensed under the Creative Commons Attribution International License (CC BY 4.0).

http://creativecommons.org/licenses/by/4.0/ (c) (i) Open Access

\begin{abstract}
Background: There is conflicting information about whether Scottish children follow the UK government recommendation of consuming 5 portions of fruit and vegetable $(\mathrm{F} \& \mathrm{~V})$ a day, or whether increased intake of $\mathrm{F} \& \mathrm{~V}$ promotes improved health. Objective: This study aimed to 1) perform a cross-sectional study of the number of $\mathrm{F} \& \mathrm{~V}$ portions that primary school children consume in relation to age ( 4 - 13 years old) and sex, 2) establish the relationship between $\mathrm{F}$ \& V intake and health parameters. Methods: Data were collected from 466 children using a specific F \& V intake questionnaire. Health parameters (weight, height, blood pressure, waist circumference, hip circumference and lung function) were measured from all of the children. Results: Children consumed on average a total of 4.50 (SD 1.86) portions of F \& V per day. F \& V intake was not significantly different between girls and boys $\left(\mathrm{t}_{(464)}=-0.397, \mathrm{P}=0.69\right)$. Children had greater intake of $\mathrm{F} \& \mathrm{~V}$ on week day compared to weekend day $\left(\mathrm{t}_{(465)}=6.42\right.$, $\mathrm{P} \leq 0.001$ ). $42.2 \%$ children achieved the recommended $\mathrm{F} \& \mathrm{~V}$ intake of five or more portions per day. After adjusting for age, sex and school, children who consumed more F \& V had several markers of positive health including greater height, and parameters reflecting pulmonary function (FEV1 and FVC) were improved, while body weight, body fat, systolic blood pressure, pulse, waist circumference, hip circumference, waist to hip and waist to height ratio were all reduced. Conclusion: The recommended level of F \& V or more can promote health among primary age children.
\end{abstract}

\section{Keywords}

Fruit Intake, Vegetable Intake, Health Parameters, Children, School

\section{Introduction}

F \& V are an essential part of a balanced diet and healthy lifestyle. They are rich 
in essential nutrients that are believed to promote good health [1]. F \& V contain high amounts of vitamins, minerals, fiber and phytochemicals and are rich in antioxidants such as Vitamin C [1]. The WHO report an insufficient intake of F $\& \mathrm{~V}$ is one of the 10 top leading risk factors for global mortality. The report suggests that increasing the intake of $\mathrm{F} \& \mathrm{~V}$ as recommended could help in preventing chronic disease and saving people's lives on average 2.7 million people annually [2]. Adequate consumption of $\mathrm{F} \& \mathrm{~V}$ reduces the risk of cardiovascular diseases, certain cancers, type 2 diabetes and obesity [2]. Moreover, "eating a variety of vegetables and fruits clearly ensures an adequate intake of most micronutrients, dietary fibers and a host of essential non-nutrient substances" [3].

Eating more than seven portions a day of F \& V for adults to derive additional health benefits with all-cause mortality and preventing chronic disease was suggested by the University College London (UCL) in 2014 following a study which looked at $>65,000$ adults in England over the years 2001-2008 [4]. It was established in the same study that vegetables show greater health benefits than fruit, with reduced deaths from cancer compared with fruit [4]. Incidences of being overweight in the long term could be prevented by of the consumption of whole fruits, which comprise a high water content, low energy density, as well as a high content of dietary fiber [5]. However, it is currently unclear whether there are significant benefits from increasing $\mathrm{F} \& \mathrm{~V}$ intake among children [6].

The current recommendation in the $\mathrm{UK}$, is to eat a variety of 5 portions of $\mathrm{F} \&$ $\mathrm{V}$ for adults and children (adults: $80 \mathrm{~g}$ each-equal to $400 \mathrm{~g} / \mathrm{d}$ ),) excluding potatoes (starchy food). For children it is suggested that a child's handful be used to count as one portion of fruit or vegetables [7]. Pulses, beans and a $150 \mathrm{ml}$ portion of fresh fruit juice each only count as one portion per day [1]. Most children of primary age fail to meet the level of F \& V recommended by the WHO in many countries [8]-[16].

The average F \& V intake for UK primary school children has been estimated at 3 - 4 portions per day [17] [18]. Older children, aged $5-12$ years old had the lowest daily intake of F \& V (2.7 portions) in comparison with younger children aged 2 - 4 years old (3.1 portions) [19]. Despite $63 \%$ of boys and $73 \%$ of girls (aged 11 - 15) being aware of the five portions per day recommendation [20], only $11 \%$ of children aged between 5 - 15 met this recommended level in 2012 [19]. This appears to be increased in younger children as in England (Leeds) children aged 3 - 7 years were found to eat an average of 2.4 items of fruit (including fruit juice; or 1.6 excluding fruit juice) and 1.6 items of vegetables per day [21]. However, up to $30 \%$ of children reportedly do not eat any fruit or vegetables on a daily basis [11]-[21]. While some reports revealed that children consumed more fruit than vegetables [10] [22] [23], others found no differences with equivalent intakes of F \& V [15] [24] [25]. In addition to age, sex was also found to impact F \& V intake with girls eating more fruit than boys [24] [25] [26]. Family affluence may have an impact on children's healthy behaviors, in that the more affluent the child's family is, the more intake of F \& V among their children [26] [27]. 
While many studies report the benefits of increased F \& V intake, the impact on specific health parameters is unclear [13]. Studies have shown a significant correlation between increased intakes of $\mathrm{F} \& \mathrm{~V}$ in intervention studies and weight loss and these interventions have been shown to work in adults [28] [29] [30]. Reductions in body mass index (BMI) were suggested to be due to increased fruit intake and decreased fat intake among the 5th grade children in the intervention group [31]. Increased intake of vegetables, however, did not have a significant effect on BMI [31]. This was suggested to be because children generally had a greater liking and intake for fruit, leading to higher fruit consumption after a school based intervention programme.

The increased incidence of obesity in children with higher intakes of energydense food and lower F \& V consumption is often suggested to be causal [32]. However not all reports on F \& V intake suggest increased intake is beneficial, and concerns about a higher consumption of fruit juices leading to weight gain among children have been raised [13] [33]. There was a strong positive association between consuming five F \& V a day and Brazilian adolescents (age 12 - 19) being overweight [25]. In contrast obese/overweight children in Philadelphia consumed fewer servings of $\mathrm{F} \& \mathrm{~V}$ in comparison with children's with normal weight (4.0 vs 7.2 servings/day) [15].

There is also conflicting evidence for other health parameters. For example, it is widely believed that dietary intake of F \& V is inversely related with blood pressure, and may prevent the development of hypertension [34]. However, other studies did not show any significant association [35] [36]. The potential mechanism behind such a relationship is also unclear [37] [38] [39]. In addition, many cross-sectional studies have shown that $\mathrm{F} \& \mathrm{~V}$ intake is correlated with improved lung function in children, which may be related to the high content of Vitamin C [40] [41] [42]. F \& V are considered to have a rich content of antioxidants, like Vitamin C; and if taken in large amounts can lead to better lung functioning as antioxidants modulate the adverse effect of ozone $\left(\mathrm{O}_{3}\right)$ on lung function as well as the resulting airway inflammatory response [41]. Importantly, a 2015 study found that antioxidants can raise the spread of cancer cells only if the patients with cancer are mice, but not in healthy subjects; however this has not yet been fully confirmed, as the testing was carried out in mice [43]. The effect of F \& V intake on health parameters is therefore still confused, and there is a need for further research in children to elucidate the impact of $F \& V$ intake on pediatric health.

The primary aim of the current study was to perform a cross-sectional survey of Scottish primary school children aged 4-13, to assess the intake of F \& V, testing for any effect of age or sex. The relationship between F \& V intake and various health related parameters was also investigated. Health parameters included (weight $(\mathrm{W})$, height $(\mathrm{H})$, body composition $(\mathrm{BC})$, systolic and diastolic blood pressure (SBP, DBP), resting pulse $(\mathrm{P})$, lung function parameters, waist circumference (WC), hip circumference (HC), waist to hip ratio (WHR) and waist to height ratio (WHtR)). 


\section{Materials and Methods}

\subsection{Subjects}

A total of 466 subjects aged $4-13$ years old (216 boys and 250 girls) participated in this study. Data were collected from seventeen participating primary schools in Aberdeenshire, Scotland.

\subsection{Fruit and Vegetables Intake Questionnaire (FVIQ)}

All F \& V intake was assessed using a questionnaire that specifically focused on the consumption of $\mathrm{F} \& \mathrm{~V}$ (Appendix). Pictures of $\mathrm{F} \& \mathrm{~V}$, shown as a child's handful, were provided in the questionnaire to provide a more precise estimation of portion quantity to aid completion of the questionnaire. The questionnaire was tested and refined by reviewers prior to distribution. The questionnaire consisted of two parts:

1) A F \& V intake diary for one week day and one weekend day. F \& V intake was categorized into five groups: fruit intake, vegetable intake, juice intake, food containing fruit and food containing vegetables. Examples of food containing fruit included pies, processed fruit snacks, and fruit yogurts and food containing vegetables included pasta sauces, soup, and vegetable stir fry. Children's parents were asked to report any fruit or vegetables (excluding potatoes) consumed in 3 main meals (breakfast, lunch, dinner) and 2 snacks (before lunch, dinner) for the 2 days. For estimating the portion sizes of liquids/other types of food (e.g. juice, soup, and stew) in the questionnaire, household measures were defined by photographs of a small glass or bowl. For food containing either fruit or vegetables the raters had to make an informed judgment on the portion size.

2) General demographic questions. Demographic information was collected including the child's date of birth, sex, number of people living in the household (adults and children), full postcode, educational level of parents and employment status. They were completed by the children's parents on their behalf. In addition parents were asked for their opinion on barriers to consuming the recommended portion of $\mathrm{F} \& \mathrm{~V}$ by their children. The $\mathrm{F} \& \mathrm{~V}$ barriers data were obtained from the same questionnaire.

\subsection{Health Parameters}

Health paremeters were measured at school. These measurements were made with the child wearing standard school uniform with shoes, hair accessories and socks or tights that would influence measurements, removed. Data were anonymized and stored in secure premises at University of Aberdeen. Some of the health parameter data was invalid due to failure to complete the measurements or refusal by the child to be measured. Height was measured to the nearest 0.1 $\mathrm{cm}$ using a standard stadiometer (SECA 217), weight and body composition were measured to the nearest $0.1 \mathrm{~kg}$ using bioelectrical impedance analyser (BIA) specifically designed for measurements in children up to 17 years old (Tanita BF-689). It depends on bioelectrical impedance analysis technique which is 
used a safe, low-level electrical signal that is passed through the body to measure fat percentage in the body. BMI was calculated, BMI $=$ Weight $(\mathrm{kg}) /$ Height $\left(\mathrm{m}^{2}\right)$. BMI in children is not an accurate measure of adiposity due to differences in growth curves between boys and girls over different ages, therefore BMI-percentile was used. Measures were adjusted for the age and sex of the child. BMIpercentile was determined and calculated online based on British 1990 (UK90) Growth Reference charts, which was developed by the UK-NHS [44] [45]. Weight classification was based on BMI-percentile cut off values as underweight (BMI: $\leq 2$ nd percentile), normal weight (BMI: $>2-<85$ th percentile), overweight (BMI: $\geq 85$ th percentile), or obese (BMI: $\geq 95$ th percentile). Waist and hip circumferences were measured to the nearest $0.1 \mathrm{~cm}$ using a tape measure (SECA Ergonomic Circumference Measuring Tape). Waist circumference was measured at the narrowest point of a normal waist (between the lowest rib and iliac crest) and hip circumference was measured at the widest area of the buttocks. Blood pressure was measured using an automatic digital B.P. monitor (Omron M10IT), for older children age 9-13 years old, and an automatic digital B.P. monitor (Omron HEM-907) for younger children age 4-8 years old. Lung function was assessed by a digital spirometer (MIR Spirobank USB Spirometer). The following measures of lung function were recorded for each child: forced expiratory volume in one second (FEV1): the volume of air that can be forcibly breathed out in one second; forced vital capacity (FVC): the total volume of air that can be forcibly breathed out in one breath; (FEV1/FVC): FEV1 as a proportion of FVC; forced expiratory flow $25 \%$ to $75 \%$ (FEF25\% - 75\%): The average rate of flow between the $25 \%$ and $75 \%$ volume point of a FVC. The lung function value $\mathrm{Z}$ scores were computed based on height, age, sex and ethnicity of children using the Global Lungs Initiative (GLI) equations [46].

\subsection{Children's Family Socioeconomic Status}

Children's parents were asked about their current address (street number and post code). Post codes and street addresses was used to determine the value of the property as an indication of socioeconomic status of a family using the Scotland based council tax bands system which categorises all property into eight bands (A-H). The council tax bands for the homes of the participants are public domain data and were obtained from Aberdeen City Council [47]. These bands were clustered into three groups low (A to $\mathrm{C}$ ), moderate ( $\mathrm{D}$ to $\mathrm{E}$ ) and high (F to $\mathrm{H})$, and also replaced by a number ( 1 - 7 for council tax bands A-G) respectively for calculating the mean for each school individually which were used to assess if there is any relationship between children's consumption of $\mathrm{F} \& \mathrm{~V}$ and socioeconomic status.

\subsection{Statistical Analysis}

Data from the questionnaires and HP measurements were coded, using Microsoft Excel, and imported later to SPSS, version 22. The average weekend and week day F \& V intakes were determined and used in all analysis. Data were 
examined for normality before analysis using the Shapiro-Wilks test ( $\mathrm{p}$-value > 0.05 ) and visually by a histogram and Q-Q plot. Log transformations were performed where necessary prior to analysis to attain normality. Measurements only were excluded if they had an incomplete test such as insufficient exhalation in the spirometry test. All parameters in the study were reported as mean, standard deviation, maximum, minimum, and the total percentage of some variables. Paired $t$-tests were used to test for differences between mean F \& V intake measured over the weekend and week days. Two-way ANOVA was used to compare mean difference between independent variables ( $F \& V$ intake, sex or age) on dependent variable (such as weight) or if there was an interaction/effect between two independent variables on dependent variable. A Tukey post-hoc test was used to determine which means are significantly different from each other when significant differences were found by ANOVA. Multiple regression analysis was performed with given health parameters as the dependent variables and several independent variables (F \& V intake, age, sex and schools) to establish a model that predicted any changes in the health markers to increased F \& V intake. A significant level of 0.05 was used.

\section{Results}

\subsection{General Characteristics of Pupils}

The general characteristics of pupils are shown in Table 1. Based on children's BMI-percentile the majority were classified as normal weight (82.8\%). In total, (6.9\%) of children were obese, $(7.3 \%)$ were overweight, $(2.8 \%)$ were underweight and for $0.2 \%$ the data were missing due to children exerting their right not to participate in the measurement.

\subsection{Demographic and Socio-Economic Characteristics}

The majority of households consisted of 2 adults (86\%) and 2 children (52.3\%). Children from Aberdeen City and Aberdeenshire lived in property values based on the council tax bandings as follows; Low (36.5\%), moderate (34.1\%) and high (21.7\%) indicating that most children lived in less affluent areas. For approximately $7.1 \%$ of children we failed to determine their postcode. In total $93.5 \%$ of children's fathers and $81.3 \%$ of mothers were employed. The highest proportion of fathers (38.5\%) had obtained college level education, with (36.6\%) attending University, while (32.0\%) of mothers had completed a college education with the highest proportion attending University (49.6\%). Demographic characteristics of the participants are shown in Table 2.

\subsection{Parent's Perspective of F \& V Intake}

A large percentage (94.2\%) of parents were aware of the government initiative (5-a-day) for consuming F \& V with, the majority rating 5-a-day as "Just right" (86\%). Despite this, only $56.6 \%$ of parents thought that 5 or more portions of $F$ \& V should be eaten daily by their children. 236 of the parents were asked to re- 
Table 1. General health characteristics of pupils participating in F \& V study.

\begin{tabular}{|c|c|c|c|c|c|c|c|}
\hline & $\mathbf{N}$ & Boys & $\mathbf{N}$ & Girls & $\mathbf{N}$ & Overall & \\
\hline Characteristic & & Mean \pm SD & & Mean \pm SD & & Mean \pm SD & Min - Max \\
\hline Age (decimal years) & 216 & $8.7 \pm 2.0$ & 250 & $8.5 \pm 1.9$ & 466 & $8.5 \pm 1.97$ & $4.5-13.2$ \\
\hline Weight (kg) & 216 & $30.5 \pm 9.0$ & 249 & $29.0 \pm 7.8$ & 465 & $29.6 \pm 8.41$ & $14.5-65.0$ \\
\hline Height $(\mathrm{cm})$ & 216 & $131.9 \pm 12.4$ & 250 & $130.0 \pm 12.2$ & 466 & $130.8 \pm 12.27$ & $101.0-165.0$ \\
\hline $\begin{array}{c}\text { Body composition } \\
\text { (fat \%) }\end{array}$ & 170 & $20.8 \pm 5.98$ & 204 & $21.9 \pm 5.43$ & 374 & $21.43 \pm 5.70$ & $5.00-43.70$ \\
\hline BMI-percentile (\%) & 216 & $56.6 \pm 30.0$ & 249 & $53.5 \pm 27.7$ & 465 & $54.97 \pm 28.75$ & $5.00-99.0$ \\
\hline $\begin{array}{c}\text { Systolic blood } \\
\text { pressure (mm Hg) }\end{array}$ & 201 & $105.0 \pm 10.0$ & 231 & $105.0 \pm 13.0$ & 432 & $104.8 \pm 11.65$ & $63-140$ \\
\hline $\begin{array}{c}\text { Diastolic blood } \\
\text { pressure }(\mathrm{mm} \mathrm{Hg})\end{array}$ & 202 & $65.0 \pm 10.8$ & 231 & $65.7 \pm 11.0$ & 433 & $65.36 \pm 10.89$ & $35-100$ \\
\hline Pulse (BPM) & 202 & $86.3 \pm 12.5$ & 232 & $87.3 \pm 12.6$ & 434 & $86.82 \pm 12.53$ & $45-122$ \\
\hline $\begin{array}{l}\text { Waist circumference } \\
\qquad(\mathrm{cm})\end{array}$ & 172 & $58.9 \pm 7.6$ & 211 & $56.6 \pm 6.5$ & 383 & $57.59 \pm 7.08$ & 89 \\
\hline $\begin{array}{l}\text { Hip Circumference } \\
(\mathrm{cm})\end{array}$ & 173 & $66.7 \pm 8.9$ & 212 & $66.4 \pm 8.2$ & 385 & $66.54 \pm 8.52$ & $48-94$ \\
\hline $\begin{array}{l}\text { Waist to Hip ratio } \\
\text { (\%) }\end{array}$ & 172 & $0.88 \pm 0.05$ & 211 & $0.85 \pm 0.05$ & 383 & $0.86 \pm 0.51$ & $0.67-0.96$ \\
\hline $\begin{array}{l}\text { Waist to Height ratio } \\
\text { (\%) }\end{array}$ & 172 & $0.45 \pm 0.04$ & 210 & $0.43 \pm 0.04$ & 382 & $0.44 \pm 0.41$ & $0.33-0.58$ \\
\hline FEV1 (Z-score)* & 185 & $-0.18 \pm 1.54$ & 203 & $-0.13 \pm 1.54$ & 388 & $-0.16 \pm 1.54$ & $-3.62-4.49$ \\
\hline FVC (Z-score)* & 186 & $0.42 \pm 2.19$ & 210 & $0.80 \pm 2.25$ & 396 & $0.62 \pm 2.23$ & $-3.55-8.14$ \\
\hline FEV1/FVC (Z-score)* & 186 & $-0.66 \pm 1.33$ & 215 & $-1.03 \pm 1.31$ & 401 & $-0.86 \pm 1.33$ & $-3.8-2.20$ \\
\hline FEF2575 (Z-score)* & 181 & $-0.60 \pm 0.89$ & 201 & $-0.91 \pm 0.90$ & 382 & $-0.76 \pm 0.91$ & $-3.15-1.41$ \\
\hline
\end{tabular}

BMI-percentile, Body Mass Index-expressed as a percentile relative to child's age and gender, mm Hg, millimeter of mercury, BPM, beat per minute, FEV1, forced expiratory volume in 1 second, L, FVC, forced vital capacity, FEV1/FVC, proportion of forced expiratory volume in 1 second and forced vital capacity, FEF2575, forced expiratory flow (25 - 75\%), Z-score *, statistical measurement of a score's relationship to the mean in a group of scores.

port on the barriers for not having more portions of $\mathrm{F} \& \mathrm{~V}$ in their child's diet. The highest barriers was the child does not like the taste of F \& V $(n=106$, $44.92 \%)$, spoil too quickly ( $\mathrm{n}=58,24.58 \%)$ and cost too much $(\mathrm{n}=55,23.31 \%)$, (Figure 1(a)). Perceived barriers differed dependent on the council tax bands for children's family (Figure 1(b)).

\subsection{Children's F \& V Intake}

The average F \& V intake was $4.50 \pm 1.86$ portions per day. No significant differences in $\mathrm{F} \& \mathrm{~V}$ intake were found between girls and boys $\left(\mathrm{t}_{(464)}=-0.397, \mathrm{p}=\right.$ $0.69)$. There were also no sex differences in both fruit intake $\left(t_{(464)}=0.19, \mathrm{p}=\right.$ $0.85)$ and vegetable intake $\left(\mathrm{t}_{(464)}=-1.06, \mathrm{p}=0.29\right)$. No significant sex differences were found in juice intake (any 100\% fresh fruit/vegetables juice), food containing fruit and vegetable intake (any food that contains fruit such as pies or vegetables such as soup) ( $p>0.05)$. Tables $3(a)-(c)$ respectively, show the average 
Table 2. Demographic characteristics of pupils participating in F \& V study by $\mathrm{n}$ and \%.

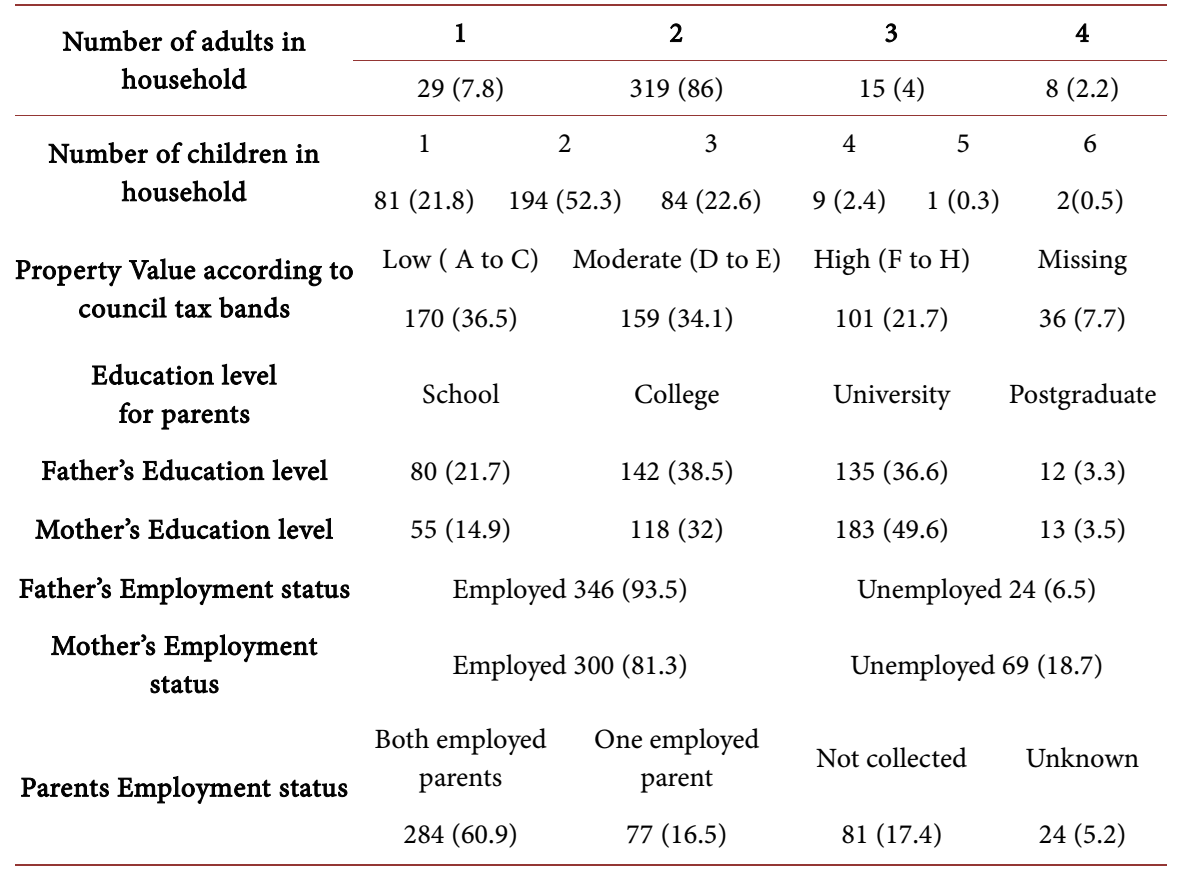

results from the combined 2 days of recording and separate week day and weekend day for F \& V intake for boys, girls and combined. 197 (42.2\%) children out of 466 achieved the recommended F \& V intake of greater than or equal to 5 portions per day.

Children had a higher F \& V intake, fruit intake, vegetable intake and juice intake during the week day than weekend day (Paired T-test: total: $\mathrm{t}_{(465)}=-6.42$, $\mathrm{p} \leq 0.001$, fruit intake: $\mathrm{t}_{(465)}=-5.93, \mathrm{p} \leq 0.001$, vegetable intake: $\mathrm{t}_{(464)}=-2.15, \mathrm{p}=$ 0.032 , juice intake: $\mathrm{t}_{(465)}=-2.76, \mathrm{p}=0.006$. There was no significant difference in week day intake $\left(\mathrm{t}_{(464)}=-0.805, \mathrm{p}=0.42\right)$ or weekend day intake $\left(\mathrm{t}_{(464)}=0.109, \mathrm{p}\right.$ $=0.91$ ) between boys and girls. There was no association between children's age and their intake of $\mathrm{F} \& \mathrm{~V}$ over total two days $\left(\mathrm{F}_{(3,462)}=1.295, \mathrm{p}=0.27\right)$ or either week day $\left(\mathrm{F}_{(3,462)}=1.571, \mathrm{p}=0.19\right)$ or weekend day $\left(\mathrm{F}_{(4,462)}=0.635, \mathrm{p}=0.59\right)$. There was no significant interaction between age and sex on intake over both week day $\left(\mathrm{F}_{(3,458)}=0.947, \mathrm{p}=0.41\right)$ or weekend day $\left(\mathrm{F}_{(3,458)}=0.972, \mathrm{p}=0.40\right)$. When intake was separated into different components there were a significant age differences in fruit intake during week day $\left(\mathrm{F}_{(3,462)}=3.027, \mathrm{p}=0.02\right)$. Posthoc Tukey tests indicated that children aged 6-8 years old had significantly higher fruit intake during week day $(2.55 \pm 1.74)$ than children aged 8-10 years old $(2.03 \pm 1.24)$. However, no age differences was found in fruit intake at the weekend $\left(\mathrm{F}_{(3,462)}=2.531, \mathrm{p}=0.08\right)$. No sex differences in fruit intake were found in week day; $\left(\mathrm{t}_{(464)}=-0.593, \mathrm{p}=0.55\right)$, or weekend day; $\left(\mathrm{t}_{(464)}=0.999, \mathrm{p}=0.32\right)$.

The same was true of vegetable intake as there were no significant age differences during week day $\left(\mathrm{F}_{(3,461)}=0.446, \mathrm{p}=0.72\right)$ or weekend day $\left(\mathrm{F}_{(3,462)}=0.447\right.$, $\mathrm{p}=0.72$ ) or between boys and girls (week day; $\mathrm{t}_{(463)}=-0.931, \mathrm{p}=0.35$, weekend day; $\mathrm{t}_{(464)}=-0.780, \mathrm{p}=0.43$ ). The difference in the mean intake of $\mathrm{F} \& \mathrm{~V}$ for week day and weekend day is shown in Figure 2. 


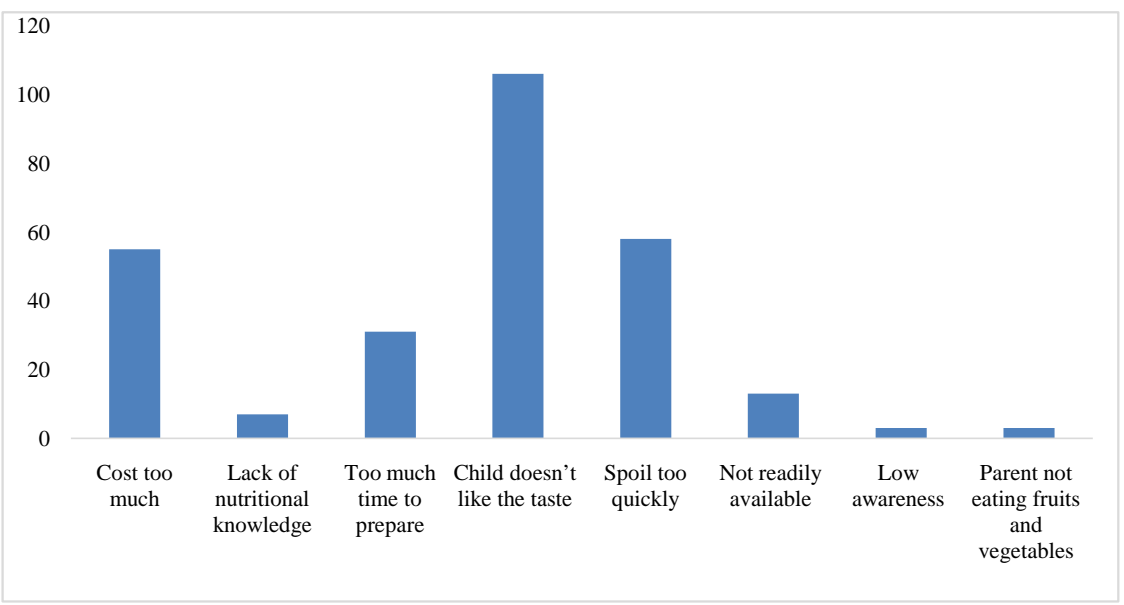

(a)

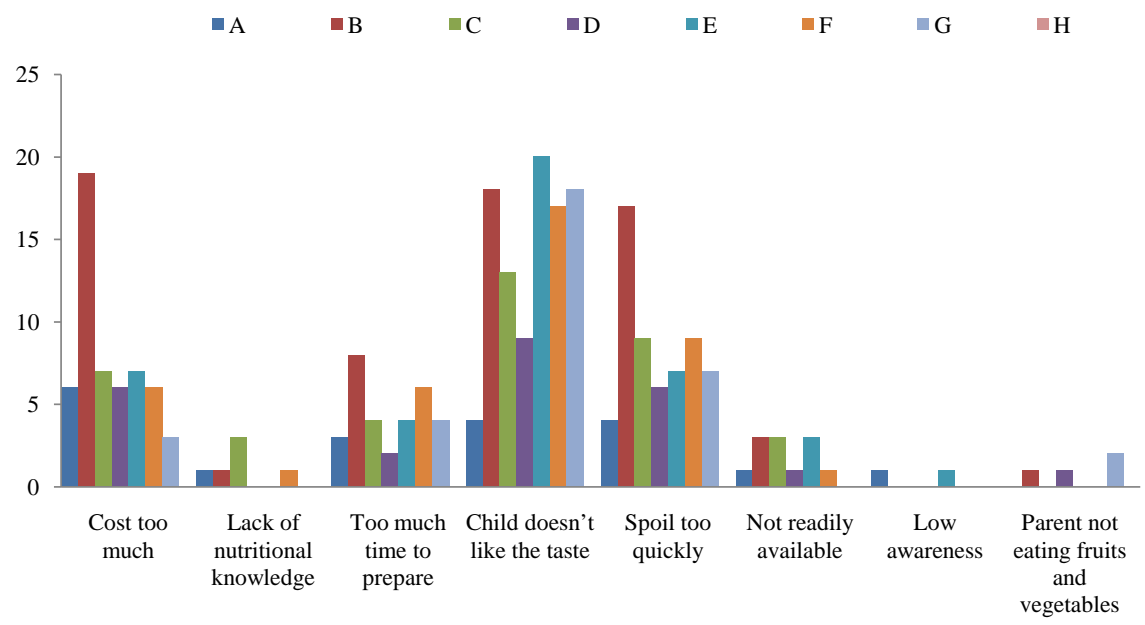

(b)

Figure 1. (a) Parents views on the general barriers for not having more portions of $F \& V$ in their child's diet; (b) Parents views on the barriers for not having more portions of F \& $\mathrm{V}$ in their child's diet according to socioeconomic status (council tax bands A to H: with A lowest).

\subsection{The Differences in F \& V Intake between Schools}

Significant differences in F \& V intake were found between schools (One-way ANOVA: $\left.\mathrm{F}_{(16,449)}=2.941, \mathrm{p}<0.001\right)$. The average over all schools ranged from consumption of 2.50 portions \pm 2.31 to 6.13 portions \pm 2.45 a day. The socioeconomic status of children's family (based on council tax bands at each school) had a statistical positive relationship with mean children's intake of $\mathrm{F} \& \mathrm{~V}$ ( $\mathrm{Li}$ near regression: $\left.\mathrm{F}_{(1,15)}=4.843, \mathrm{p}=0.044, \mathrm{r}^{2}=0.24\right)$, (Figure 3 ).

\subsection{Effects of Parental Education/Employment Status}

Maternal education level had no significant association with children's total F \& $\mathrm{V}$ intake, on either week or weekend days (One-way ANOVA: total; $\mathrm{F}_{(3,365)}=$ $0.68, \mathrm{p}=0.56$, week day; $\mathrm{F}_{(3.365)}=0.63, \mathrm{p}=0.59$, weekend day; $\mathrm{F}_{(3,365)}=0.48, \mathrm{p}=$ 0.69). Likewise father's education did not show any significant correlation with 
Table 3. (a) Average daily F \& V intake recorded over two days in primary school children; (b) F \& V on Week day in boys and girls; (c) F \& V over weekend day in boys and girls.

(a)

\begin{tabular}{cccc}
\hline Sex & $\begin{array}{c}\text { Boys } \\
(\mathbf{n}=\mathbf{2 1 6})\end{array}$ & $\begin{array}{c}\text { Girls } \\
(\mathbf{n}=\mathbf{2 5 0})\end{array}$ & $\begin{array}{c}\text { Overall } \\
(\mathbf{n}=\mathbf{4 6 6})\end{array}$ \\
\hline Characteristic & Mean \pm SD & Mean \pm SD & Mean \pm SD \\
Total F \& V (\#/day) & $4.46 \pm 1.76$ & $4.52 \pm 1.94$ & $4.50 \pm 1.86$ \\
Vegetable (\#/day) & $1.43 \pm 1.01$ & $1.53 \pm 1.02$ & $1.49 \pm 1.02$ \\
Fruit (\#/day) & $2.04 \pm 1.15$ & $2.02 \pm 1.26$ & $2.03 \pm 1.21$ \\
Juice (\#/day) & $0.58 \pm 0.60$ & $0.61 \pm 0.64$ & $0.59 \pm 062$ \\
Fruit food (\#/day) & $0.17 \pm 0.33$ & $0.14 \pm 0.33$ & $0.15 \pm 0.33$ \\
Vegetable food (\#/day) & $0.23 \pm 0.41$ & $0.21 \pm 0.33$ & $0.22 \pm 0.37$
\end{tabular}

(b)

\begin{tabular}{cccc}
\hline Sex & $\begin{array}{c}\text { Boys } \\
(\mathrm{n}=216)\end{array}$ & $\begin{array}{c}\text { Girls } \\
(\mathrm{n}=250)\end{array}$ & $\begin{array}{c}\text { Overall } \\
(\mathrm{n}=466)\end{array}$ \\
\hline Characteristic & Mean \pm SD & Mean \pm SD & Mean \pm SD \\
Total F \& V (\#/day) & $4.18 \pm 2.05$ & $4.17 \pm 2.24$ & $4.17 \pm 2.16$ \\
Vegetable (\#/day) & $1.37 \pm 1.23$ & $1.46 \pm 1.29$ & $1.41 \pm 1.26$ \\
Fruit (\#/day) & $1.89 \pm 1.32$ & $1.76 \pm 1.37$ & $1.82 \pm 1.35$ \\
Juice (\#/day) & $0.53 \pm 0.66$ & $0.56 \pm 0.72$ & $0.55 \pm 0.69$ \\
Fruit food (\#/day) & $0.13 \pm 0.35$ & $0.12 \pm 0.39$ & $0.12 \pm 0.37$ \\
Vegetable food (\#/day) & $0.25 \pm 0.54$ & $0.24 \pm 0.51$ & $0.25 \pm 0.52$ \\
\hline
\end{tabular}

(c)

\begin{tabular}{cccc}
\hline Sex & $\begin{array}{c}\text { Boys } \\
(\mathbf{n}=\mathbf{2 1 6})\end{array}$ & $\begin{array}{c}\text { Girls } \\
(\mathbf{n}=\mathbf{2 5 0})\end{array}$ & $\begin{array}{c}\text { Overall } \\
(\mathbf{n}=\mathbf{4 6 6})\end{array}$ \\
\hline Characteristic & Mean \pm SD & Mean \pm SD & Mean \pm SD \\
Total F \& V (\#/day) & $4.73 \pm 2.14$ & $4.88 \pm 2.11$ & $4.81 \pm 2.12$ \\
Vegetable (\#/day) & $1.50 \pm 1.19$ & $1.60 \pm 1.19$ & $1.56 \pm 1.19$ \\
Fruit (\#/day) & $2.19 \pm 1.42$ & $2.27 \pm 1.54$ & $2.23 \pm 1.48$ \\
Juice (\#/day) & $0.62 \pm 0.73$ & $0.66 \pm 0.74$ & $0.64 \pm 0.73$ \\
Fruit food (\#/day) & $0.20 \pm 0.46$ & $0.16 \pm 0.43$ & $0.18 \pm 0.44$ \\
Vegetable food (\#/day) & $0.20 \pm 0.47$ & $0.18 \pm 0.39$ & $0.19 \pm 0.43$ \\
\hline
\end{tabular}

children's intake on total F \& V intake and weekend day (One-way ANOVA: total; $\mathrm{F}_{(3,365)}=2.41, \mathrm{p}=0.06$, weekend day; $\left.\mathrm{F}_{(3,365)}=2.03, \mathrm{p}=0.10\right)$ but there was a marginally significant effect on week day; $\left(\mathrm{F}_{(3.365)}=2.91, \mathrm{p}=0.03\right)$. Post-hoc Tukey tests indicated that children of university educated fathers had significantly higher intake of week day F \& V $(5.34 \pm 2.15, \mathrm{p}=0.028)$ than children whose fathers highest level of education was high school $(4.50 \pm 2.06, \mathrm{p}=0.028)$.

Children of employed mothers had higher week day F \& V intake (Two-sample-T-test: week day; $\left.\mathrm{t}_{(366)}=-2.18, \mathrm{p}=0.03\right)$ but not for their total and weekend day F \& V intake (Two-sample-T-test: total; $\mathrm{t}_{(366)}=-1.39, \mathrm{p}=0.16$, weekend day; 


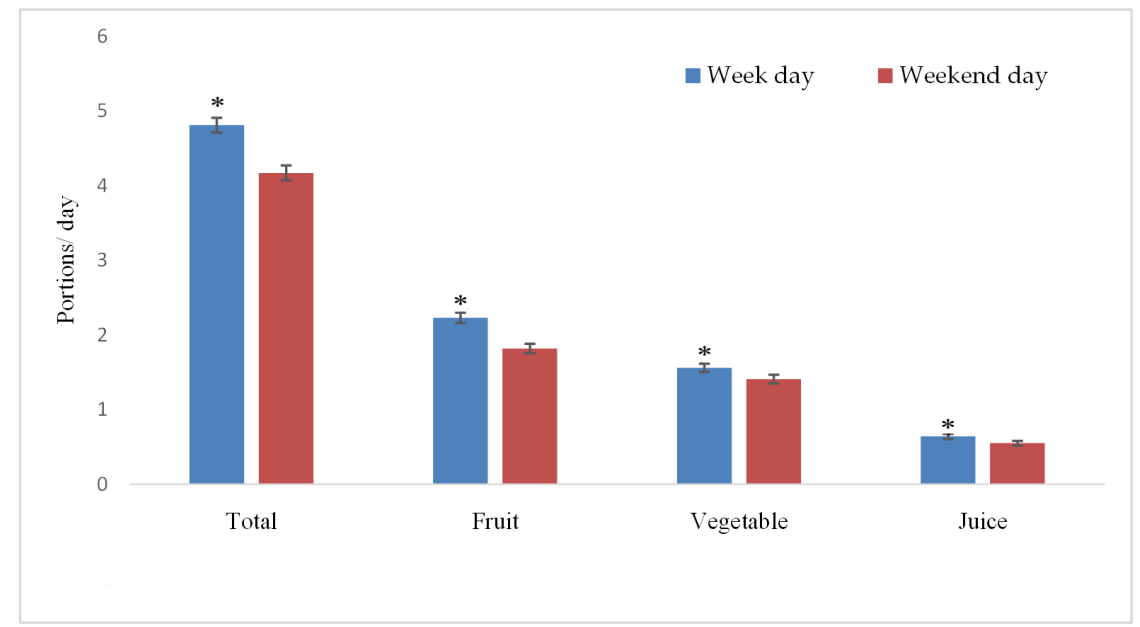

Figure 2. F \& V intake on week day and weekend day. $(M \pm S E)$ Asterisks denote significant differences.

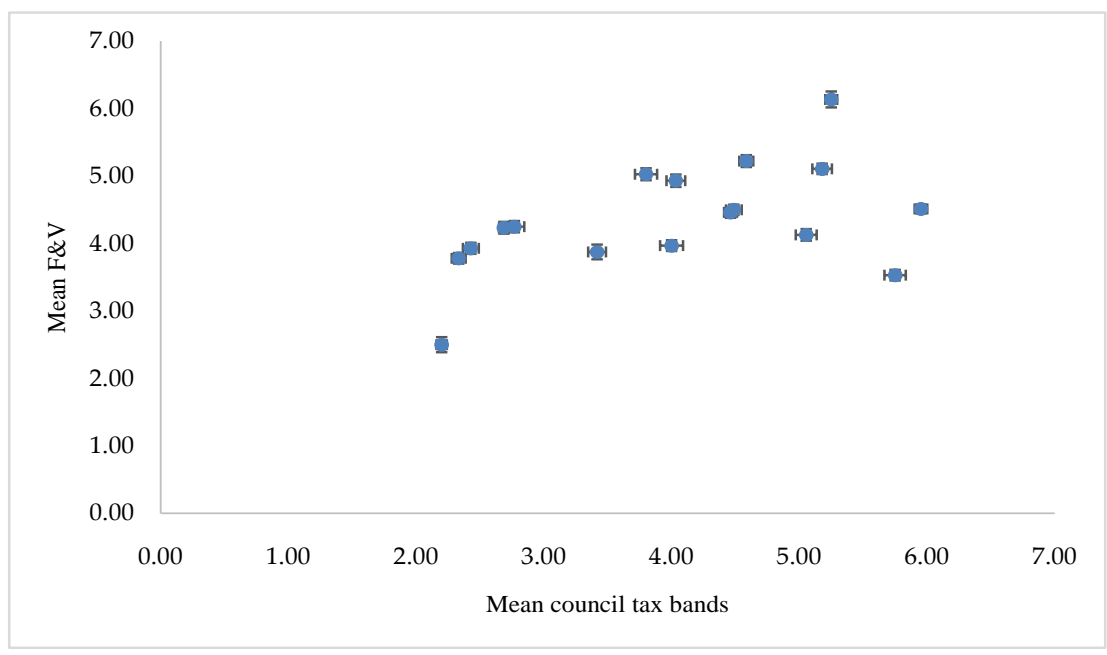

Figure 3. Scatter plot of the relationship between the mean of F \& V intake and council tax bands at each schools with \pm SE.

$\left.\mathrm{t}_{(366)}=-0.23, \mathrm{p}=0.81\right)$. Father's employment status showed no association with children's intake for total, week day and weekend day (Two-sample-T-test: total; $\mathrm{t}_{(368)}=-0.77, \mathrm{p}=0.44$, week day; $\mathrm{t}_{(368)}=-1.26, \mathrm{p}=0.20$, weekend day; $\mathrm{t}_{(368)}=$ $-0.07, \mathrm{p}=0.94)$. Children whose parents were both employed had higher week day F \& V intake $(5.10 \pm 2.12)$ in comparison with children who had only parent in employment $(4.38 \pm 2.14)$, (Two-sample-T-test: week day; $\mathrm{t}_{(359)}=2.63, \mathrm{p}=$ 0.009 ).

No differences were found in children's total and weekend day F \& V intake (Two-sample-T-test: total; $\mathrm{t}_{(359)}=1.82, \mathrm{p}=0.07$, weekend day; $\mathrm{t}_{(359)}=0.53, \mathrm{p}=$ $0.59)$ whose parents were both employed.

\subsection{Association between Property Value as an Indication of Socioeconomic Status and Children's Intake of F \& V}

There was a significant association between high council tax bands (council tax 
band: F-G-H) and total as well as weekend day intakes of F \& V in children (total: $\mathrm{F}_{(2,427)}=4.60, \mathrm{p}=0.010$, weekend day: $\left.\mathrm{F}_{(2,427)}=4.41, \mathrm{p}=0.01\right)$. However, children's week day F \& V intake was not associated with the value of the property where the children lived (week day: $\mathrm{F}_{(2,427)}=2.52, \mathrm{p}=0.08$ ).

Post-hoc Tukey tests indicated that F \& V intake during both week and weekend days in children living in high property value (council tax band: F-G$\mathrm{H})$ were significantly higher $(4.91 \pm 2.01, \mathrm{p}=0.008)$ in comparison with children living in low property value (council tax: A-B-C) $(4.23 \pm 1.76)$. After examining the weekend day separately, Post-hoc Tukey tests indicated that children living in high value properties (council tax band: F-G-H) had significantly higher intakes than those living in low value properties on weekend days $(4.63 \pm 2.40, \mathrm{p}=$ $0.011)(3.86 \pm 1.87)$.

\subsection{The Association between Children's F \& V Intake and Number of Children/Adults Household}

Total, week day and weekend day F \& V were not significantly related to the number of children in the household (One Way ANOVA total: $\mathrm{F}_{(4,366)}=1.21, \mathrm{p}=$ 0.30 , week day: $\mathrm{F}_{(4,366)}=1.60, \mathrm{p}=0.17$, weekend day: $\left.\mathrm{F}_{(4,366)}=0.43, \mathrm{p}=0.78\right)$. When examining the relationship separately with fruit intake and vegetable intake, there was no significant association found for total, week day and weekend day ( $p>0.05$ for all comparisons). Likewise, there was no significant association found when assessing the relationship of total F \& V intake, fruit intake and vegetable intake with number of adults in the house ( $p>0.05$ for all comparisons).

\subsection{The Association between F \& V Intake and HP}

A multiple regression analysis was used to determine if there was any association between the level of F \& V intake and markers of health after adjusting for sex, age and school. Multiple regression analysis results for total F \& V intake, fruit intake and vegetable intake are presented in Table 4.

All four variables ( $F \& \mathrm{~V}$, age, sex and school) added significantly to the prediction, $\mathrm{p}<0.05$. Children that consumed more $\mathrm{F} \& \mathrm{~V}$ were taller $\left(\mathrm{F}_{(4,461)}=\right.$ 170.996, $\left.\mathrm{p}<0.001, \mathrm{r}^{2}=0.53\right)$ and had improved forced expiratory volume in one second (FEV1-Z) $\left(\mathrm{F}_{(4,383)}=3.411, \mathrm{p}=0.009, \mathrm{r}^{2}=0.03\right)$ and forced vital capacity $($ FVC_Z $)\left(\mathrm{F}_{4,391)}=9.677, \mathrm{p}<0.001, \mathrm{r}^{2}=0.09\right)$. Other significantly improved health markers related to $\mathrm{F} \& \mathrm{~V}$ intake included reduced body weight $\left(\mathrm{F}_{(4,460)}=\right.$ 91.071, $\left.\mathrm{p}<0.001, \mathrm{r}^{2}=0.44\right)$, reduced body fat $\left(\mathrm{F}_{4,369)}=3.464, \mathrm{p}=0.009, \mathrm{r}^{2}=\right.$ $0.03)$, systolic blood pressure $\left(\mathrm{F}_{4,427)}=5.739, \mathrm{p}<0.001, \mathrm{r}^{2}=0.05\right)$, pulse $\left(\mathrm{F}_{(4,429)}\right.$ $\left.=6.721, \mathrm{p}<0.001, \mathrm{r}^{2}=0.05\right)$, waist circumference $\left(\mathrm{F}_{(4,378)}=39.401, \mathrm{p}<0.001, \mathrm{r}^{2}\right.$ $=0.29)$, hip circumference $\left(\mathrm{F}_{(4,380)}=53.845, \mathrm{p}<0.001, \mathrm{r}^{2}=0.36\right)$, WHR $\left(\mathrm{F}_{(4,378)}=\right.$ 3.195, $\left.\mathrm{p}=0.013, \mathrm{r}^{2}=0.03\right)$ and WHtR $\left(\mathrm{F}_{(4,377)}=10.652, \mathrm{p}<0.001, \mathrm{r}^{2}=0.10\right)$. There was however a significant reduction in the total volume of air that can be forcibly expired in one breath (FEV1/FVC-Z) $\left(\mathrm{F}_{(4,396)}=4.295, \mathrm{p}=0.002, \mathrm{r}^{2}=\right.$ $0.04)$ and forced expiratory flow $25 \%$ to $75 \%$ (FEF25-75_Z) $\left(\mathrm{F}_{(4,377)}=5.717, \mathrm{p}<\right.$ $\left.0.001, \mathrm{r}^{2}=0.05\right)$.

When examined separately, children who consumed more fruit were taller 
Table 4. Multiple regression analysis results for total F \& V intake combined or individually are presented.

\begin{tabular}{|c|c|c|}
\hline $\mathrm{HP}$ versus total F \& V & R-Squared & $\mathrm{P}$-value \\
\hline Weight $(\mathrm{kg})$ & 0.442 & $<0.001$ \\
\hline Height $(\mathrm{cm})$ & 0.539 & $<0.001$ \\
\hline Body composition (\%) & 0.036 & 0.009 \\
\hline BMI-percentile (\%) & 0.013 & 0.193 \\
\hline SYS BP (mm Hg) & 0.051 & $<0.001$ \\
\hline DIAS BP $(\mathrm{mm} \mathrm{Hg})$ & 0.013 & 0.243 \\
\hline Pulse (bpm) & 0.059 & $<0.001$ \\
\hline Waist circumference $(\mathrm{cm})$ & 0.294 & $<0.001$ \\
\hline Hip Circumference $(\mathrm{cm})$ & 0.362 & $<0.001$ \\
\hline Waist to Hip ratio (\%) & 0.033 & 0.013 \\
\hline Waist to Height ratio (\%) & 0.102 & $<0.001$ \\
\hline FEV1 (Z-score) $)^{*}$ & 0.034 & 0.009 \\
\hline FVC $(\mathrm{Z} \text {-score })^{*}$ & 0.090 & $<0.001$ \\
\hline FEV1/FVC (Z-score) ${ }^{*}$ & 0.042 & 0.002 \\
\hline FEF2575 (Z-score) $)^{*}$ & 0.057 & $<0.001$ \\
\hline HP versus fruit intake & R-Squared & $P$-value \\
\hline Weight $(\mathrm{kg})$ & 0.442 & $<0.001$ \\
\hline Height $(\mathrm{cm})$ & 0.596 & $<0.001$ \\
\hline Body composition (\%) & 0.033 & 0.015 \\
\hline BMI-percentile (\%) & 0.009 & 0.389 \\
\hline SYS BP (mm Hg) & 0.050 & $<0.001$ \\
\hline DIAS BP (mm Hg) & 0.012 & 0.285 \\
\hline Pulse(bpm) & 0.056 & $<0.001$ \\
\hline Waist circumference $(\mathrm{cm})$ & 0.294 & $<0.001$ \\
\hline Hip Circumference $(\mathrm{cm})$ & 0.360 & $<0.001$ \\
\hline Waist to Hip ratio (\%) & 0.100 & $<0.001$ \\
\hline Waist to Height ratio (\%) & 0.140 & $<0.001$ \\
\hline FEV1 (Z-score) ${ }^{*}$ & 0.034 & 0.009 \\
\hline FVC $(Z \text {-score })^{*}$ & 0.093 & $<0.001$ \\
\hline FEV1/FVC (Z-score $)^{*}$ & 0.037 & 0.004 \\
\hline FEF2575 (Z-score) ${ }^{*}$ & 0.060 & $<0.001$ \\
\hline HP versus vegetable intake & R-Squared & P-value \\
\hline Weight $(\mathrm{kg})$ & 0.442 & $<0.001$ \\
\hline Height $(\mathrm{cm})$ & 0.596 & $<0.001$ \\
\hline Body composition (\%) & 0.035 & 0.011 \\
\hline BMI-percentile (\%) & 0.010 & 0.345 \\
\hline
\end{tabular}


Continued

\begin{tabular}{ccc}
\hline SYS BP (mm Hg) & 0.053 & $<0.001$ \\
DIAS BP (mm Hg) & 0.016 & 0.130 \\
Pulse(bpm) & 0.072 & $<0.001$ \\
Waist circumference (cm) & 0.299 & $<0.001$ \\
Hip Circumference (cm) & 0.369 & $<0.001$ \\
Waist to Hip ratio (\%) & 0.032 & 0.014 \\
Waist to Height ratio (\%) & 0.111 & $<0.001$ \\
FEV1 (Z-score) & 0.035 & 0.009 \\
FVC (Z-score) & 0.092 & $<0.001$ \\
FEV1/FVC (Z-score) & 0.044 & 0.001 \\
FEF2575 (Z-score) & 0.054 & $<0.001$ \\
\hline
\end{tabular}

Z-score ${ }^{\star}$, statistical measurement of a score's relationship to the mean in a group of scores.

$\left(\mathrm{F}_{(4,461)}=170.366, \mathrm{p}<0.001, \mathrm{r}^{2}=0.59\right)$, and had increased waist circumference $\left(\mathrm{F}_{4,378)}=39.390, \mathrm{p}<0.001, \mathrm{r}^{2}=0.29\right)$, WHR $\left(\mathrm{F}_{(4,377)}=10.420, \mathrm{p}<0.001, \mathrm{r}^{2}=\right.$ $0.10)$ and WHtR $\left(\mathrm{F}_{(4,377)}=10.420, \mathrm{p}<0.001, \mathrm{r}^{2}=0.10\right)$, while also having a reduced weight $\left(\mathrm{F}_{(4,460)}=91.147, \mathrm{p}<0.001, \mathrm{r}^{2}=0.44\right)$, reduced body fat $\left(\mathrm{F}_{(4,369)}=\right.$ $\left.3.109, \mathrm{p}=0.015, \mathrm{r}^{2}=0.03\right)$, systolic blood pressure $\left(\mathrm{F}_{(4,427)}=5.610, \mathrm{p}<0.001, \mathrm{r}^{2}=\right.$ $0.05)$, pulse $\left(\mathrm{F}_{(4,429)}=6.357, \mathrm{p}<0.001, \mathrm{r}^{2}=0.05\right)$, hip circumference $\left(\mathrm{F}_{(4,380)}=\right.$ 53.437, $\left.\mathrm{p}<0.001, \mathrm{r}^{2}=0.36\right)$, FVC_Z $\left(\mathrm{F}_{(4,391)}=10.023, \mathrm{p}<0.001, \mathrm{r}^{2}=0.09\right)$, FEV1-Z $\left(\mathrm{F}(4,383)=3.410, \mathrm{p}=0.009, \mathrm{r}^{2}=0.03\right), \mathrm{FEV} 1 / \mathrm{FVC}-\mathrm{Z}\left(\mathrm{F}_{(4,396)}=3.847, \mathrm{p}\right.$ $\left.=0.004, \mathrm{r}^{2}=0.03\right)$ and FEF2575_Z $\left(\mathrm{F}_{(4,377)}=5.968, \mathrm{p}<0.001, \mathrm{r}^{2}=0.06\right)$.

When the association was examined for only vegetable intake, there was a significant correlation with increased height $\left(\mathrm{F}_{(4,461)}=170.198, \mathrm{p}<0.001, \mathrm{r}^{2}=0.59\right)$, FEV1-Z $\left(\mathrm{F}_{(4,383)}=3.431, \mathrm{p}=0.009, \mathrm{r}^{2}=0.03\right)$, and FVC_Z $\left(\mathrm{F}_{(4,391)}=9.885, \mathrm{p}<\right.$ $\left.0.001, \mathrm{r}^{2}=0.09\right)$, and similar reductions weight $\left(\mathrm{F}_{(4,460)}=91.137, \mathrm{p}<0.001, \mathrm{r}^{2}=\right.$ $0.44)$, body composition $\left(\mathrm{F}_{4,369)}=3.340, \mathrm{p}=0.011, \mathrm{r}^{2}=0.03\right)$, systolic blood pressure $\left(\mathrm{F}_{(4,427)}=5.945, \mathrm{p}<0.001, \mathrm{r}^{2}=0.05\right)$, pulse $\left(\mathrm{F}_{(4,429)}=8.361, \mathrm{p}<0.001, \mathrm{r}^{2}\right.$ $=0.07)$, waist circumference $\left(\mathrm{F}_{(4,378)}=40.398, \mathrm{p}<0.001, \mathrm{r}^{2}=0.29\right)$, hip circumference $\left(\mathrm{F}_{(4,380)}=55.460, \mathrm{p}<0.001, \mathrm{r}^{2}=0.36\right)$, WHR $\left(\mathrm{F}_{(4,378)}=3.122, \mathrm{p}=0.015, \mathrm{r}^{2}\right.$ $=0.03), \operatorname{WHtR}\left(\mathrm{F}_{(4,377)}=11.764, \mathrm{p}<0.001, \mathrm{r}^{2}=0.11\right)$, FEV1/FVC-Z $\left(\mathrm{F}_{(4,396)}=\right.$ $\left.4.609, \mathrm{p}=0.001, \mathrm{r}^{2}=0.04\right)$ and FEF2575_Z $\left(\mathrm{F}_{(4,377)}=5.333, \mathrm{p}<0.001, \mathrm{r}^{2}=0.05\right)$. This showed that both $\mathrm{F} \& \mathrm{~V}$ intake were positively correlated with aspects of the children's health. Other HPs (notably BMI-percentile and diastolic blood pressure) did not show any significant association with total $\mathrm{F} \& \mathrm{~V}$ intake combined, or separately.

\section{Discussion}

The relationship between $\mathrm{F} \& \mathrm{~V}$ intake and health parameters was examined in primary school children in Aberdeenshire, Scotland. The results revealed that the majority (57.8\%) of children did not consume the recommended level of 5-aday as advised by the WHO [3]. This result is similar to previous UK-based stu- 
dies [21] [48]. However, with $42.2 \%$ of children in the present study eating the recommended 5 a-day, our results were higher than the 14\% reported by the Scottish Health Survey in 2 to 15 -year-olds in 2015 [49]. This difference could be because the sample used here was not demographically representative of the whole of Scotland, with Aberdeenshire being relatively affluent.

The mean intake in our study was 4.50 portions of $\mathrm{F} \& \mathrm{~V}$ per day; higher than previously published in 2012 (2.7 portions per day) and in 2014 (2.8 portions per day) which also used similar survey methods [49] [50]. Consistent with the Scottish Government report of 2015, we found no differences between boys and girls in their intake of F \& V [49].

Children had a higher intake of F \& V when combined or fruit, vegetable and juice intake individually, on the week day compared with during the Weekend day. This contrasts a recently published study showing that Czech young children, especially girls, consumed more fruit, vegetables and breakfast on weekend day [51].

A possible explanation for our finding might be related to the usual home availability of this type of food over the week day, or the child's preferences. Supporting the present data, Lachapelle and colleagues showed a higher consumption of F \& V Monday to Friday among French children compared to over the weekend day [52]. A second interpretation for the higher intake of $F \& V$ during week day may be related to the parental intake of $\mathrm{F} \& \mathrm{~V}$, as it was shown previously that parental F \& V intake correlates positively with children's F \& V intake [53], meaning that if children observed their parents consuming $\mathrm{F} \& \mathrm{~V}$ at home, they ate more. School lunches obviously have a possible impact on F \& V intake especially for those children who have lunch at school, as a balanced lunch meal containing F \& V is always available to children [54], especially of primary age, and this may reflect the higher intake during the week day more than weekend day. A food standard for school lunches was introduced by Scottish Health Survey in 2008 for improving Scottish children's diet, particularly to increase F \& V intake. "A choice of at least two types of vegetables and two types of fruit (not including fruit juice) must be provided every day as part of the school lunch." This standard, undoubtedly, with other factors (e.g. parents' encouragement), is believed to have an impact on approaching the recommended daily level by children if the levels of $\mathrm{F} \& \mathrm{~V}$ in their snacks and dinner are maintained [55].

A range of factors were expected to influence children's F \& V intake, including parental educational level, the employment status of both parents, number of adults/children per household and council tax band (socioeconomic status). The educational level of the fathers was not positively associated with total and weekend day F \& V intake in children. However, children of university educated fathers had increased week day F \& V intake, as has been observed previously [56]. Maternal education level was not related to F \& V intake, This result is in agreement with a Chinese case study in Hong Kong in 2010 among children and their mothers from around 10 primary schools [57] but contradictory to a study 
in 2014 which found that children with a high socioeconomic status (particularly highly educated mothers) ate more $\mathrm{F} \& \mathrm{~V}$ than children with low socioeconomic status (low-educated mothers) [53].

Regardless of the effect of employment status/education level on F \& V intake, socioeconomic factors are recognised to have a major impact on diet choice [58] and lower status may lead to a poor intake of healthy food, including F \& V in children. We also found that socioeconomic status (property value) was a significant factor in the present study, supporting these previous findings. It was previously reported that poor diet choices were linked to families who live in lowincome areas, and most of the healthy food, such as F \& V, were consumed by families who are higher in socioeconomic status [58] [59]. Furthermore, the current study addressed determinants for not eating the recommended level of five portions of F \& V by children in a socio-economic context (e.g. people living in deprived areas and on low income), as similar findings were previously reported in qualitative studies among adults [60], British young adults age 18-24 years old [61], among both children and adults [62], and quantitative studies of Portuguese high school students [63]. These determinants were: cost, lack of access to $\mathrm{F} \& \mathrm{~V}$, too much time to prepare, disliking the taste of $\mathrm{F} \& \mathrm{~V}$, home availability, and food preferences.

Increased intake of $\mathrm{F} \& \mathrm{~V}$ showed a correlation with improvements in many HP in our study. Studies on the relationship between F \& V and HP in children are uncommon, although information was available for adults. An increase in F \& V intake was associated with a reduction in children's weight. These results were in accordance with recent publications reporting a negative relationship between F \& V intake and body weight in women aged 20 - 60 years old [28], young-middle and older adults [64] [65] and children (7 and 15 years old) [66]. Also examining fruit on its own, (children (1 - 5 years old) [33] and adults (30 75 years old) [67] and vegetables on their own were correlated with reduced weight [67].

BMI-percentile was not associated with F \& V intake, combined or separately, and this finding was consistent with results from previous studies for $\mathrm{F} \& \mathrm{~V}$ intake and fruit intake in both children (8 - 9 years old) [68], preschool children [69], 9 - 14 years olds [13] and adults (undergraduate students) [70]. However, contradictory to our findings a Spanish study of children and adolescents age 6 18 years old found a reduction in BMI-SDS among the obese group $(\mathrm{n}=184)$ not the control group $(\mathrm{n}=185)$ with total dietary antioxidant capacity (TAC). BMI was also previously reported to be inversely related to only vegetable intake in children and adolescents [13] which was inconsistent with the lack of association found here.

Although we found no association between BMI and F \& V intake there was a significant association with body fat. In a previous study of adults, it was reported that increased $\mathrm{F} \& \mathrm{~V}$ intake, in addition to calorie reduction, aided loss of body fat and weight among individuals aged 19 - 50, and this result was based on serum carotenoid concentrations (a biomarker of F \& V intake) [71]. Results 
from two studies conducted in 2010 and 2014 in children confirmed a negative association between only vegetable intake [72] or food high in antioxidants e.g. F \& V (with only obese children) [66] and body fat. Studies to determine the association between increasing $\mathrm{F} \& \mathrm{~V}$ intake and losing body fat among children possibly is still under examination by others. Similar results revealed that waist circumference [73], hip circumference, WHtR and WHR [66] were all negatively associated with increased F \& V intake. These effects were not previously reported in studies where these parameters were measured for example in young women aged 18 - 28 years [74] or in preschool children 3-6 years old [69]. However, increased in WC, WHR and WHtR was observed with increased fruit intake which was not expected. The separate and opposite associations of vegetable intake and fruit intake with WC, WHR and WHtR requires confirmation.

Consistent with several recent studies for adults and children, systolic blood pressure [37] [38] [39] [61] [64] [75] and pulse rate (heart rate) [66] were correlated with greater intake of vegetables and fruit. No change was observed in diastolic blood pressure with increasing F \& V intake combined or individual in this study, which was unexpected but may have occurred because the current $\mathrm{F}$ \& V intake among our participants was not reaching the recommended level or above to promote positive health benefits. The relationship between the pulse, diastolic blood pressure, and children's intake of F \& V is still unclear, and more research related to this area is needed. Better pulmonary function (FEV1, FVC) was significantly correlated with F \& V intake combined or separately with only vegetable intake. Much research has investigated this relationship, with the majority agreeing that combined $\mathrm{F} \& \mathrm{~V}$ intake, particularly citrus fruits, is positively correlated with lung function (FEV1-FVC) among adults in both a cohort study [76] and a cross-sectional study [77] [78], as well as among children in a crosssectional study [40], and a cohort study [42].

However, low fruit intake was associated with reductions in all of the parameters of pulmonary function, and these findings were similar to those reported previously in children [41], where they have been ascribed to lower intake of antioxidant vitamins (C, E, A) (which were examined by food frequency question based on nutrients intake estimation between diet with/without supplementation). The negative significant (defects) result in some of the pulmonary function related to F \& V combined or separately (FEV1\%-FEF2575) perhaps because our participants had less intake of antioxidant vitamins which were not examined in our FVIQ. These vitamins are mostly obtained from diet (e.g. including F \& V) or supplementation. It showed how it is positively had pulmonary function improvements on studies conducted in the UK among adults 18 70 years old [79] and adolescents 11 - 19 years old [41].

\section{Conclusion}

Children we studied had high intakes of $\mathrm{F} \& \mathrm{~V}$ relative to previous surveys in the UK but most still did not meet the recommendation of 5 a day. With a positive correlation between health related parameters and increased F \& V intake, this 
suggests that promotion of $\mathrm{F} \& \mathrm{~V}$ is warranted, although we should always bear in mind this was a cross sectional study and associations do not prove causality. In addition, given some of the associations were negative; the links are complex and need further study. While the benefits of increasing F \& V are well documented after children reach adolescence and adulthood there is relatively little information regarding impacts on children.

\section{Acknowledgements}

The authors are grateful to all schools, parents and children who participated in this study. Thank you to Sarah Brady who participated in data collection. Thank you to Dr. Catherine Hambly, Dr. Sharon E. Mitchell and Prof. John R. Speakman for their comments and suggestions.

\section{Authors' Contributions}

The literature search, data collection and statistical analyses were performed by HA. The original manuscript was written by HA. MC assisted in data collection.

\section{Ethics Approval and Consent to Participate}

Ethical approval was obtained by University of Aberdeen-Ethics Review Board of the College of Life Sciences and Medicine (CERB/2014/12/1123). Written consent forms were collected from the children's parents/guardians and children provided verbal consent on the day of measurements.

\section{References}

[1] Slavin, J.L. and Lloyd, B. (2012) Health Benefits of Fruits and Vegetables. Advances in Nutrition, 3, 506-516. https://doi.org/10.3945/an.112.002154

[2] World Health Organization (2016) Fruit and Vegetable Promotion Initiative, a Meeting Report 2003.

http://www.who.int/dietphysicalactivity/publications/f\&v_promotion_initiative_rep ort.pdf

[3] World Health Organization (2016) Promoting Fruit and Vegetable Consumption around the World 2003. http://www.who.int/dietphysicalactivity/fruit/en/

[4] Oyebode, O., Gordon-Dseagu, V., Walker, A. and Mindell, J.S. (2014) Fruit and Vegetable Consumption and All-Cause, Cancer and CVD Mortality: Analysis of Health Survey for England Data. Journal of Epidemiology and Community Health, 68, 856-862. https://doi.org/10.1136/jech-2013-203500

[5] Tetens, I. and Alinia, S. (2009) The Role of Fruit Consumption in the Prevention of Obesity. Journal of Horticultural Science and Biotechnology, 84, 47-51. https://doi.org/10.1080/14620316.2009.11512594

[6] Ledoux, T.A., Hingle, M.D. and Baranowski, T. (2011) Relationship of Fruit and Vegetable Intake with Adiposity: A Systematic Review. Obesity Reviews, 12, e143150. https://doi.org/10.1111/j.1467-789X.2010.00786.x

[7] National Health Service (NHS) (2016) 5 A DAY Portion Sizes-How Much Is a Portion of Fruit or Veg. http://www.nhs.uk/change4life/pages/five-a-day-portion-sizes.aspx

[8] Ganann, R., Fitzpatrick-Lewis, D., Ciliska, D. and Peirson, L. (2012) Communi- 
ty-Based Interventions for Enhancing Access to Or Consumption of Fruit and Vegetables among Five to 18-Year Olds: A Scoping Review. BMC Public Health, 12, 711. https://doi.org/10.1186/1471-2458-12-711

[9] Attorp, A., Scott, J.E., Yew, A.C., Rhodes, R.E., Barr, S.I. and Naylor, P.J. (2014) Associations between Socioeconomic, Parental and Home Environment Factors and Fruit and Vegetable Consumption of Children in Grades Five and Six in British Columbia, Canada. BMC Public Health, 14, 150. https://doi.org/10.1186/1471-2458-14-150

[10] Blanchette, L. and Brug, J. (2005) Determinants of Fruit and Vegetable Consumption among 6-12-Year-Old Children and Effective Interventions to Increase Consumption. Journal of Human Nutrition and Dietetics, 18, 431-443. https://doi.org/10.1111/j.1365-277X.2005.00648.x

[11] Christian, M.S., Evans, C.E., Nykjaer, C., Hancock, N. and Cade, J.E. (2014) Evaluation of the Impact of a School Gardening Intervention on Children's Fruit and Vegetable Intake: A Randomised Controlled Trial. International Journal of Behavioral Nutrition and Physical Activity, 11, 99. https://doi.org/10.1186/s12966-014-0099-7

[12] Dennison, B.A., Rockwell, H.L. and Baker, S.L. (1998) Fruit and Vegetable Intake in Young Children. Journal of the American College of Nutrition, 17, 371-378. https://doi.org/10.1080/07315724.1998.10718778

[13] Field, A.E., Gillman, M.W., Rosner, B., Rockett, H.R. and Colditz, G.A. (2003) Association between Fruit and Vegetable Intake and Change in Body Mass Index among a Large Sample of Children and Adolescents in the United States. International Journal of Obesity and Related Metabolic Disorders, 27, 821-826. https://doi.org/10.1038/sj.ijo.0802297

[14] Kim, S.A., Moore, L.V., Galuska, D., Wright, A.P., Harris, D., Grummer-Strawn, L.M., Merlo, C.L., Nihiser, A.J., Rhodes, D.G., Division of Nutrition, P.A., et al. (2014) Vital Signs: Fruit and Vegetable Intake among Children-United States, 2003-2010. Morbidity and Mortality Weekly Report, 63, 671-676.

[15] Miller, P., Moore, R.H. and Kral, T.V. (2011) Children's Daily Fruit and Vegetable Intake: Associations with Maternal Intake and Child Weight Status. Journal of $\mathrm{Nu}$ trition Education and Behavior, 43, 396-400. https://doi.org/10.1016/j.jneb.2010.10.003

[16] Nielsen, S.J., Rossen, L.M., Harris, D.M. and Odgen, C.L. (2014) Fruit and Vegetable Consumption of U.S. Youth, 2009-2010. NCHS Data Brief, 159, 1-8.

[17] Henderson, L., Gregory, J. and Swan, G. (2002) National Diet and Nutrition Survey: Adults Aged 19 to 64 Years. Volume 1, Types and Quantities of Foods Consumed. The National Diet and Nutrition Survey: Adults Aged 19 to 64 Years, 1, 1-88.

[18] Ransley, J.K., Greenwood, D.C., Cade, J.E., Blenkinsop, S., Schagen, I., Teeman, D., Scott, E., White, G. and Schagen, S. (2007) Does the School Fruit and Vegetable Scheme Improve Children's Diet? A Non-Randomised Controlled Trial. Journal of Epidemiology and Community Health, 61, 699-703.

https://doi.org/10.1136/jech.2006.052696

[19] The Scottish Government (2016) The Scottish Health Survey 2012: Volume 1: Main Report: 5 Diet. http://www.gov.scot/Publications/2013/09/3684/9

[20] Health Survey for England (2016) Healthy Lifestyles: Knowledge, Attitudes and Behavior. http://www.hscic.gov.uk/pubs/hse07healthylifestyles

[21] Cade, J.E., Frear, L. and Greenwood, D.C. (2006) Assessment of Diet in Young Children with an Emphasis on Fruit and Vegetable Intake: Using Cadet-Child and Diet Evaluation Tool. Public Health Nutrition, 9, 501-508. https://doi.org/10.1079/PHN2005871 
[22] Mak, T.N., Prynne, C.J., Cole, D., Fitt, E., Roberts, C., Bates, B. and Stephen, A.M. (2012) Assessing Eating Context and Fruit and Vegetable Consumption in Children: New Methods Using Food Diaries in the UK National Diet and Nutrition Survey Rolling Programme. International Journal of Behavioral Nutrition and Physical Activity, 9, 126. https://doi.org/10.1186/1479-5868-9-126

[23] Ramsay, S.A., Eskelsen, A.K., Branen, L.J., Armstrong Shultz, J. and Plumb, J. (2014) Nutrient Intake and Consumption of Fruit and Vegetables in Young Children. Infant, Child, and Adolescent Nutrition, 6, 332-344. https://doi.org/10.1177/1941406414549622

[24] Wind, M., de Bourdeaudhuij, I., te Velde, S.J., Sandvik, C., Due, P., Klepp, K.I. and Brug, J. (2006) Correlates of Fruit And Vegetable Consumption among 11-year-old Belgian-Flemish and Dutch Schoolchildren. Journal of Nutrition Education and Behavior, 38, 211-221. https://doi.org/10.1016/j.jneb.2006.02.011

[25] Rieth, M.A., Moreira, M.B., Fuchs, F.D., Moreira, L.B. and Fuchs, S.C. (2012) Fruits and Vegetables Intake and Characteristics Associated among Adolescents from Southern Brazil. Nutrition Journal, 11, 95. https://doi.org/10.1186/1475-2891-11-95

[26] Zarnowiecki, D., Ball, K., Parletta, N. and Dollman, J. (2014) Describing Socioeconomic Gradients in Children's Diets-Does the Socioeconomic Indicator Used Matter? International Journal of Behavioral Nutrition and Physical Activity 2014, 11, 44. https://doi.org/10.1186/1479-5868-11-44

[27] Moore, G.F. and Littlecott, H.J. (2015) School- and Family-Level Socioeconomic Status and Health Behaviors: Multilevel Analysis of a National Survey in Wales, United Kingdom. Journal of School Health, 85, 267-275. https://doi.org/10.1111/josh.12242

[28] Ello-Martin, J.A., Roe, L.S., Ledikwe, J.H., Beach, A.M. and Rolls, B.J. (2007) Dietary Energy Density in the Treatment of Obesity: A Year-Long Trial Comparing 2 Weight-Loss Diets. American Journal of Clinical Nutrition, 85, 1465-1477.

[29] Epstein, L.H., Gordy, C.C., Raynor, H.A., Beddome, M., Kilanowski, C.K. and Paluch, R. (2001) Increasing Fruit and Vegetable Intake and Decreasing Fat and Sugar Intake in Families at Risk for Childhood Obesity. Obesity Research, 9, 171-178. https://doi.org/10.1038/oby.2001.18

[30] Shenoy, S.F., Poston, W.S., Reeves, R.S., Kazaks, A.G., Holt, R.R., Keen, C.L., Chen, H.J., Haddock, C.K., Winters, B.L., Khoo, C.S., et al. (2010) Weight Loss in Individuals with Metabolic Syndrome Given Dash Diet Counseling When Provided a Low Sodium Vegetable Juice: A Randomized Controlled Trial. Nutrition Journal, 9, 8. https://doi.org/10.1186/1475-2891-9-8

[31] Angelopoulos, P.D., Milionis, H.J., Grammatikaki, E., Moschonis, G. and Manios, Y. (2009) Changes in BMI and Blood Pressure after a School Based Intervention: The Children Study. European Journal of Public Health, 19, 319-325. https://doi.org/10.1093/eurpub/ckp004

[32] Lorson, B.A., Melgar-Quinonez, H.R. and Taylor, C.A. (2009) Correlates of Fruit and Vegetable Intakes in US Children. Journal of the American Dietetic Association, 109, 474-478. https://doi.org/10.1016/j.jada.2008.11.022

[33] Faith, M.S., Dennison, B.A., Edmunds, L.S. and Stratton, H.H. (2006) Fruit Juice Intake Predicts Increased Adiposity Gain in Children from Low-Income Families: Weight Status-by-Environment Interaction. Pediatrics, 118, 2066-2075. https://doi.org/10.1542/peds.2006-1117

[34] Shi, L., Krupp, D. and Remer, T. (2014) Salt, Fruit and Vegetable Consumption and Blood Pressure Development: A Longitudinal Investigation in Healthy Children. British Journal of Nutrition, 111, 662-671. 
https://doi.org/10.1017/S0007114513002961

[35] Gomes Bda, M. and Alves, J.G. (2009) Prevalence of High Blood Pressure and Associated Factors in Students from Public Schools in Greater Metropolitan Recife, Pernambuco State, Brazil, 2006. Cadernos de Saúde Pública, 25, 375-381.

[36] Singh, A.K., Maheshwari, A., Sharma, N. and Anand, K. (2006) Lifestyle Associated Risk Factors in Adolescents. Indian Journal of Pediatrics, 73, 901-906. https://doi.org/10.1007/BF02859283

[37] Couch, S.C. and Daniels, S.R. (2005) Diet and Blood Pressure in Children. Current Opinion in Pediatrics, 17, 642-647. https://doi.org/10.1097/01.mop.0000172817.87261.4f

[38] Damasceno, M.M., de Araujo, M.F., de Freitas, R.W., de Almeida, P.C. and Zanetti, M.L. (2011) The Association between Blood Pressure in Adolescents and the Consumption of Fruits, Vegetables and Fruit Juice-An Exploratory Study. Journal of Clinical Nursing, 20, 1553-1560. https://doi.org/10.1111/j.1365-2702.2010.03608.x

[39] Moore, L.L., Bradlee, M.L., Singer, M.R., Qureshi, M.M., Buendia, J.R. and Daniels, S.R. (2012) Dietary Approaches to Stop Hypertension (Dash) Eating Pattern and Risk of Elevated Blood Pressure in Adolescent Girls. British Journal of Nutrition, 108, 1678-1685. https://doi.org/10.1017/S000711451100715X

[40] Cook, D.G., Carey, I.M., Whincup, P.H., Papacosta, O., Chirico, S., Bruckdorfer, K.R. and Walker, M. (1997) Effect of Fresh Fruit Consumption on Lung Function and Wheeze in Children. Thorax, 52, 628-633. https://doi.org/10.1136/thx.52.7.628

[41] Gilliland, F.D., Berhane, K.T., Li, Y.F., Gauderman, W.J., McConnell, R. and Peters, J. (2003) Children's Lung Function and Antioxidant Vitamin, Fruit, Juice, and Vegetable Intake. American Journal of Epidemiology, 158, 576-584.

https://doi.org/10.1093/aje/kwg181

[42] Romieu, I., Barraza-Villarreal, A., Escamilla-Nunez, C., Texcalac-Sangrador, J.L., Hernandez-Cadena, L., Diaz-Sanchez, D., De Batlle, J. and Del Rio-Navarro, B.E. (2009) Dietary Intake, Lung Function and Airway Inflammation in Mexico City School Children Exposed to Air Pollutants. Respiratory Research, 10, 122. https://doi.org/10.1186/1465-9921-10-122

[43] Piskounova, E., Agathocleous, M., Murphy, M.M., Hu, Z., Huddlestun, S.E., Zhao, Z., Leitch, A.M., Johnson, T.M., DeBerardinis, R.J. and Morrison, S.J. (2015) Oxidative Stress Inhibits Distant Metastasis by Human Melanoma Cells. Nature, 527, 186-191. https://doi.org/10.1038/nature15726

[44] Cole, T.J., Freeman, J.V. and Preece, M.A. (1995) Body Mass Index Reference Curves for the UK, 1990. Archives of Disease in Childhood, 73, 25-29. https://doi.org/10.1136/adc.73.1.25

[45] National Health Service (NHS) (2016) BMI Healthy Weight Calculator. http://www.nhs.uk/Tools/Pages/Healthyweightcalculator.aspx

[46] Quanjer, P.H., Stanojevic, S., Cole, T.J., Baur, X., Hall, G.L., Culver, B.H., Enright, P.L., Hankinson, J.L., Ip, M.S., Zheng, J., et al. (2012) Multi-Ethnic Reference Values for Spirometry for the 3-95-Yr Age Range: The Global Lung Function 2012 Equations. European Respiratory Journal, 40, 1324-1343.

https://doi.org/10.1183/09031936.00080312

[47] Aberdeen City Council (2016) Band Search. https://ecitizen.aberdeencity.gov.uk/publicaccesslive/selfservice/services/counciltax/ bandsearch.htm

[48] Christian, M.S., Evans, C.E., Hancock, N., Nykjaer, C. and Cade, J.E. (2013) Family Meals Can Help Children Reach Their 5 a Day: A Cross-Sectional Survey of Children's Dietary Intake from London Primary Schools. Journal of Epidemiology and 
Community Health, 67, 332-338. https://doi.org/10.1136/jech-2012-201604

[49] The Scottish Government (2016) The Scottish Health Survey 2014: Volume 1: Main Report: 4 Diet. http://www.gov.scot/Publications/2015/09/6648/318775

[50] The Scottish Government (2016) The Scottish Health Survey 2011: Volume 2Children: 3 Diet. http://www.gov.scot/Publications/2012/09/3327/29

[51] Voracova, J., Sigmund, E., Sigmundova, D. and Kalman, M. (2015) Changes in Eating Behaviours among Czech Children and Adolescents from 2002 to 2014 (HBSC Study). International Journal of Environmental Research and Public Health, 12, 15888-15899. https://doi.org/10.3390/ijerph121215028

[52] Lachapelle, D., Gamache, C., Brodeur, J.M. and Sevigny, J. (1989) Frequency of Food Consumption in Children. Weekdays versus Weekends. Journal of the Canadian Dental Association, 55, 61-65.

[53] Van Ansem, W.J., Schrijvers, C.T., Rodenburg, G. and van de Mheen, D. (2014) Maternal Educational Level and Children's Healthy Eating Behaviour: Role of the Home Food Environment (Cross-Sectional Results from the INPACT Study). International Journal of Behavioral Nutrition and Physical Activity, 11, 113. https://doi.org/10.1186/s12966-014-0113-0

[54] The Scottish Government (2016) Hungry for Success-A Whole School Approach to School Meals in Scotland. http://www.gov.scot/Publications/2003/02/16273/17571

[55] The Scottish Government (2016) Healthy Eating in Schools: A Guide to Implementing the Nutritional Requirements for Food and Drink in Schools (Scotland) Regulations 2008. http://www.gov.scot/Publications/2008/09/12090355/0

[56] Lehto, E., Ray, C., Te Velde, S., Petrova, S., Duleva, V., Krawinkel, M., Behrendt, I., Papadaki, A., Kristjansdottir, A., Thorsdottir, I., et al. (2015) Mediation of Parental Educational Level on Fruit and Vegetable Intake among Schoolchildren in Ten European Countries. Public Health Nutrition, 18, 89-99. https://doi.org/10.1017/S136898001300339X

[57] Yung, T.K., Lee, A., Ho, M.M., Keung, V.M. and Lee, J.C. (2010) Maternal Influences on Fruit and Vegetable Consumption of Schoolchildren: Case Study in Hong Kong. Maternal \& Child Nutrition, 6, 190-198.

[58] Boylan, S., Lallukka, T., Lahelma, E., Pikhart, H., Malyutina, S., Pajak, A., Kubinova, R., Bragina, O., Stepaniak, U., Gillis-Januszewska, A., et al. (2011) Socio-Economic Circumstances and Food Habits in Eastern, Central and Western European Populations. Public Health Nutrition, 14, 678-687. https://doi.org/10.1017/S1368980010002570

[59] Darmon, N. and Drewnowski, A. (2008) Does Social Class Predict Diet Quality? American Journal of Clinical Nutrition, 87, 1107-1117.

[60] John, J.H. and Ziebland, S. (2004) Reported Barriers to Eating More Fruit and Vegetables Before and After Participation in a Randomized Controlled Trial: A Qualitative Study. Health Education Research, 19, 165-174. https://doi.org/10.1093/her/cyg016

[61] Herbert, G., Kennedy, O., Lobb, A. and Butler, L. (2010) Young Adults and the 5 a Day Campaign: Perceived Benefits and Barriers of Eating More Fruits and Vegetables. International Journal of Consumer Studies, 34, 657-664. https://doi.org/10.1111/j.1470-6431.2010.00872.x

[62] Krolner, R., Rasmussen, M., Brug, J., Klepp, K.I., Wind, M. and Due, P. (2011) Determinants of Fruit and Vegetable Consumption among Children and Adolescents: A Review of the Literature. Part II: Qualitative Studies. International Journal of Behavioral Nutrition and Physical Activity, 8, 112. 
https://doi.org/10.1186/1479-5868-8-112

[63] Quintas, M., Rocha, A. and Gil, L. (2010) Promotion of Fruit and Vegetable Consumption in Children and Adolescents Through the Use of Minimally Processed Products. Acta Horticulturae, 877, 95-104. https://doi.org/10.17660/ActaHortic.2010.877.6

[64] Mathanghi, S.K., Hemalatha, G., Amutha, S. and Arulmozhiselvan, S. (2015) Impact of Fruit and Vegetable Intake by Healthy Subjects on the Risk Factors of Cardiovascular Diseases. The Indian Journal of Nutrition and Dietetics, 52, 80-87.

[65] Aljadani, H.M., Patterson, A., Sibbritt, D., Hutchesson, M.J., Jensen, M.E. and Collins, C.E. (2013) Diet Quality, Measured by Fruit and Vegetable Intake, Predicts Weight Change in Young Women. Journal of Obesity, 2013, 525161. https://doi.org/10.1155/2013/525161

[66] Puchau, B., Ochoa, M.C., Zulet, M.A., Marti, A., Martinez, J.A. and Members, G. (2010) Dietary Total Antioxidant Capacity and Obesity in Children and Adolescents. International Journal of Food Sciences and Nutrition, 61, 713-721. https://doi.org/10.3109/09637481003757860

[67] Bertoia, M.L., Mukamal, K.J., Cahill, L.E., Hou, T., Ludwig, D.S., Mozaffarian, D., Willett, W.C., Hu, F.B. and Rimm, E.B. (2016) Correction: Changes in Intake of Fruits and Vegetables and Weight Change in United States Men and Women Followed for up to 24 Years: Analysis from Three Prospective Cohort Studies. PLoS Medicine, 13, e1001956. https://doi.org/10.1371/journal.pmed.1001956

[68] Roccaldo, R., Censi, L., D’Addezio, L., Toti, E., Martone, D., D’Addesa, D., Cernigliaro, A. and Group, Z.S. (2014) Adherence to the Mediterranean Diet in Italian School Children (The ZOOM8 Study). International Journal of Food Sciences and Nutrition, 65, 621-628. https://doi.org/10.3109/09637486.2013.873887

[69] De Bock, F., Breitenstein, L. and Fischer, J.E. (2012) Positive Impact of a PreSchool-Based Nutritional Intervention on Children's Fruit and Vegetable Intake: Results of a Cluster-Randomized Trial. Public Health Nutrition, 15, 466-475. https://doi.org/10.1017/S136898001100200X

[70] Reis, L.C., Correia, I.C. and Mizutani, E.S. (2014) Stages of Changes for Fruit and Vegetable Intake and Their Relation to the Nutritional Status of Undergraduate Students. Einstein (São Paulo), 12, 48-54. https://doi.org/10.1590/S1679-45082014AO2926

[71] Whigham, L.D., Valentine, A.R., Johnson, L.K., Zhang, Z., Atkinson, R.L. and Tanumihardjo, S.A. (2012) Increased Vegetable and Fruit Consumption during Weight Loss Effort Correlates with Increased Weight and Fat Loss. Nutrition \& Diabetes, 2, e48. https://doi.org/10.1038/nutd.2012.22

[72] Wang, C., Huang, L. and Butler, C. (2014) Body Weight Status Reflects the Attitudes and Behaviors Related to Vegetable Consumption among Kentucky Adults. The FASEB Journal, 28, 640.

[73] Newby, P.K., Muller, D., Hallfrisch, J., Qiao, N., Andres, R. and Tucker, K.L. (2003) Dietary Patterns and Changes in Body Mass Index and Waist Circumference in Adults. American Journal of Clinical Nutrition, 77, 1417-1425.

[74] Azadbakht, L., Haghighatdoost, F., Feizi, A. and Esmaillzadeh, A. (2013) Breakfast Eating Pattern and Its Association with Dietary Quality Indices and Anthropometric Measurements in Young Women in Isfahan. Nutrition, 29, 420-425. https://doi.org/10.1016/j.nut.2012.07.008

[75] Moore, L.L., Singer, M.R., Bradlee, M.L., Djousse, L., Proctor, M.H., Cupples, L.A. and Ellison, R.C. (2005) Intake of Fruits, Vegetables, and Dairy Products in Early Childhood and Subsequent Blood Pressure Change. Epidemiology, 16, 4-11. 
https://doi.org/10.1097/01.ede.0000147106.32027.3e

[76] Butland, B.K., Fehily, A.M. and Elwood, P.C. (2000) Diet, Lung Function, and Lung Function Decline in a Cohort of 2512 Middle Aged Men. Thorax, 55, 102-108. https://doi.org/10.1136/thorax.55.2.102

[77] Carey, I.M., Strachan, D.P. and Cook, D.G. (1998) Effects of Changes in Fresh Fruit Consumption on Ventilatory Function in Healthy British Adults. American Journal of Respiratory and Critical Care Medicine, 158, 728-733. https://doi.org/10.1164/ajrccm.158.3.9712065

[78] Garcia-Larsen, V., Amigo, H., Bustos, P., Bakolis, I. and Rona, R.J. (2015) Ventilatory Function in Young Adults and Dietary Antioxidant Intake. Nutrients, 7, 2879 2896. https://doi.org/10.3390/nu7042879

[79] Britton, J.R., Pavord, I.D., Richards, K.A., Knox, A.J., Wisniewski, A.F., Lewis, S.A., Tattersfield, A.E. and Weiss, S.T. (1995) Dietary Antioxidant Vitamin Intake and Lung Function in the General Population. American Journal of Respiratory and Critical Care Medicine, 151, 1383-1387.

https://doi.org/10.1164/ajrccm.151.5.7735589

\section{Abbreviations}

F \& V: fruit and vegetable;

FVIQ: fruit and vegetable intake questionnaire;

HP: health parameters;

BMI: body mass index;

W: weight;

BC: body composition;

SBP: systolic blood pressure;

DBP: diastolic blood pressure;

P: pulse;

WC: waist circumference;

HC: hip circumference;

WHR: waist to hip ratio;

WHtR: waist to height ratio;

FEV1-Z: forced expiratory volume in one second-standard deviation score;

FVC: forced vital capacity;

FEV1/FVC: FEV1 as a proportion of FVC;

FEF25-75\%: forced expiratory flow $25 \%$ to $75 \%$;

Z-SCORE: statistical measurement of a score's relationship to the mean in a group of scores. 


\section{Appendix}

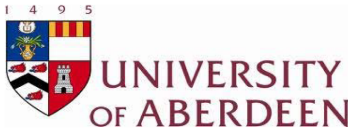

\section{FRUIT \& VEGETABLE}

\section{PROJECT}

Dear Parent,

Thank you for consenting to participate in this study on fruit and vegetable intake in school children. We have been into the school to measure the health parameters in your child and now we would like to ask you to fill out the attached food diary so that we can assess your child's fruit and vegetable intake over 2 days (preferable a week day and weekend day). Please find instructions for filling out the diary attached.

We would appreciate if you could fill out the form as completely as possible and return it to the school within the next two weeks.

If you have any questions or are unable to fill out the questionnaire at this time, please contact us by email: r01haa13@abdn.ac.uk.

Kind regards,

Heba Althubaiti

University of Aberdeen

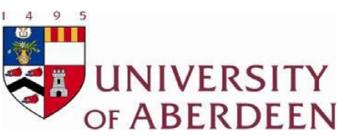

FRUIT \& VEGETABLE

OF ABERDEEN

\section{PROJECT}

Child's Code:

School Name:

Date:

\section{General questions}

This information is used only for the purpose of the study and all data will be stored anonymously.

1. What is your child's sex?

Male $\bigcirc$ Female

2. What is your child's date of birth?

3. What is your child's ethnicity?

4. How many people live at your house?

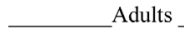
Children.

5. What is the full postcode of the child's home

6. Please indicate the educational level for child's mother and father?

$$
\begin{aligned}
& \text { Mother } \bigcirc \text { school } \bigcirc \text { College } \bigcirc \text { University } \bigcirc \text { Other ............... } \\
& \text { Father } \bigcirc \text { school } \bigcirc \text { College } \bigcirc \text { University } \bigcirc \text { Other .............. }
\end{aligned}
$$

7. What is your current status?

$$
\begin{aligned}
& \text { Mother } \bigcirc \text { Employed } \bigcirc \text { Unemployed } \\
& \text { Father } \bigcirc \text { Employed } \bigcirc \text { Unemployed }
\end{aligned}
$$

8. How many portions of fruit and vegetables do you think your child eats on an average day?

\footnotetext{
9. Have you heard about the 5-a-day fruit and vegetable campaign? $\bigcirc$ Yes $\bigcirc$ No

10. In your view are the guidelines for fruit and vegetables intake $\bigcirc$ Too high $\bigcirc$ Too low $\bigcirc$ Just right.

11. Is there any other information on your child's diet/health that you wish to tell

us?
} 


\section{Food diary}

Please keep a diary of all the fruit and vegetables your child is eating over 2 days (preferably a week and weekend day). Include all fruit and vegetables, dried fruit, fruit juice and foods containing fruit and vegetables. Please check the attached guidelines for instruction on how to fill out the diary.

Day 1
\begin{tabular}{|c|c|c|}
\hline Daily Meals & $\begin{array}{c}\text { Food description } \\
\text { (Fruit and vegetables and any food containing } \\
\text { them) }\end{array}$ & Amount \\
\hline Breakfast & & \\
\hline Snack & & \\
\hline Lunch & & \\
\hline Snack & & \\
\hline Dinner & & \\
\hline
\end{tabular}

Day 2:
\begin{tabular}{|c|c|c|}
\hline Daily Meals & $\begin{array}{c}\text { Food description } \\
\text { (Fruit and vegetables and any food containing them) }\end{array}$ & Amount \\
\hline Breakfast & & \\
\hline Snack & & \\
\hline Lunch & & \\
\hline Snack & & \\
\hline Dinner & & \\
\hline
\end{tabular}

Guidelines for filling out the food diary

Please write down all the fruit and vegetables that your child eats over 2 days (preferably try to include 1 week day and 1 weekend day).

Note the date of measurement on the diary and indicate what type of fruit and vegetables your child ate at different times of the day (breakfast, lunch, snack time etc.). If the child has not eating any fruits or vegetables at any part of the day, just fill in 'None'.

\section{What foods to include?}

- All fresh fruit and fresh vegetables

- Dried fruit and vegetables, e.g., raisins, apricots etc.

Only fresh fruit juice or smoothies.

- Food containing fruits or vegetables, e.g., blueberry muffin, fruit bar, fruit peelers, pasta sauce, yoghurt, ready meals, soups, puddings, sandwiches etc.

If you are unsure, check the packaging and if it says 1 of your 5 a day, please include it in the diary

\section{What foods not to include?}

- Please do not count potatoes because they are starchy foods (i.e., chips, mash, potato waffles etc.)

- Do not include diluting juice or fruit shoots etc.

\section{What is a portion?}

Because children of different ages have different needs, one portion of fruit or vegetables is generally measured based on their hand size. Please use a handful to indicate the portion size or use small bowl, glass as indicated in the photographs given below.

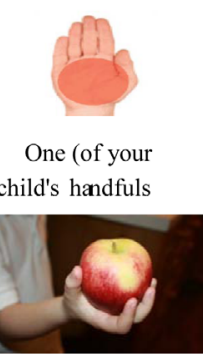

2 handfuls apple

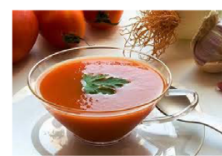

Small Bowl

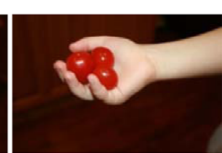

1 handful tomatoes 1 handful of raisins

Small glass

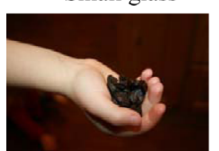

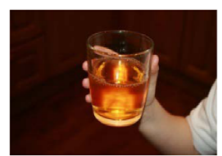

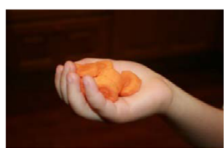

1 handful of carrots 


\section{EXAMPLE OF FILLED OUT FOOD DIARY}

Day 1

Date: _ 15 June 2015

\begin{tabular}{|l|l|l|}
\hline Daily Meals & \multicolumn{1}{|c|}{$\begin{array}{c}\text { Food description } \\
\text { (Fruit and vegetables and any food containing } \\
\text { them) }\end{array}$} & \multicolumn{1}{|c|}{ Amount } \\
\hline Breakfast & Fresh orange Juice & 1 small glass \\
\hline Snack & & 1 handful \\
\hline Lunch & $\begin{array}{l}\text { Raisins } \\
\text { Cucumber and tomato }\end{array}$ & $\begin{array}{l}\text { 2 handfuls } \\
2 \text { handfuls }\end{array}$ \\
\hline Snack & & none \\
\hline Dinner & None & 1 small bowl \\
\hline
\end{tabular}

Day 2:

Date: 20 June 2015

\begin{tabular}{|l|l|l|}
\hline Daily Meals & \multicolumn{1}{|c|}{$\begin{array}{c}\text { Food description } \\
\text { (Fruit and vegetables and any food containing }\end{array}$} & \multicolumn{1}{|c|}{ Amount } \\
\hline Breakfast & Blueberry muffin & 1 small \\
\hline Snack & None & None \\
\hline Lunch & Raisins & 1 handful \\
\hline Snack & Fresh apple juice & 1 carton (200 ml) \\
\hline Dinner & $\begin{array}{l}\text { Pasta sauce, incl. tomatoes, carrots and celery } \\
\text { Strawberry yoghurt }\end{array}$ & 2 handfuls \\
\hline
\end{tabular}


Submit or recommend next manuscript to SCIRP and we will provide best service for you:

Accepting pre-submission inquiries through Email, Facebook, LinkedIn, Twitter, etc. A wide selection of journals (inclusive of 9 subjects, more than 200 journals)

Providing 24-hour high-quality service

User-friendly online submission system

Fair and swift peer-review system

Efficient typesetting and proofreading procedure

Display of the result of downloads and visits, as well as the number of cited articles Maximum dissemination of your research work

Submit your manuscript at: http://papersubmission.scirp.org/

Or contact fns@scirp.org 